\title{
Macromycetes determined in Muradiye (Van) district
}

\section{Ant J Bot Q16}

Received : 09.12.2019 Accepted : 01.01.2020 Online : 20.03 .2020

\author{
Gözde ÇAGLí1 ${ }^{\oplus}$, Avni ÖZTÜRK² \\ ${ }^{1}$ Süphan Quarter, TEIAŞ housing, 65100, Van, Turkey \\ ${ }^{2}$ Muratpaşa Quarter, Fatih Gemalmaz housing, Yakutiye, Erzurum, Turkey \\ *gozde_1989@hotmail.com, 2avniozturk49@gmail.com
}

\section{Muradiye (Van) ilçesinde belirlenen makromantarlar}

\begin{abstract}
The study was based on macrofungi samples collected from the region within the boundaries of Muradiye district of Van province in 2014 and 2015. As a result of field and laboratory studies 86 macrofungi species belonging to 50 genera, 25 families, seven orders and three classes within the divisions Ascomycota and Basidiomycota were determined. The determined taxa are listed together with their habitats, substrates, collection locality, geographic coordinates and personel voucher numbers. One of them, Melanophyllum haematospermum (Bull.: Fr.) Kreisel, is a new record for Turkey as the first member of the genus Melanophyllum Velen.
\end{abstract}

Key words: Biodiversity, new record, macrofungi, mycota, Turkey

Özet: Çalışma 2014 ve 2015 yıllarında Van'ın Muradiye ilçe sınırları içinde kalan bölgeden toplanan makromantar örnekleri üzerinde gerçekleştirilmiştir. Arazi ve laboratuvar çalışmaları sonucunda Ascomycota ve Basidiomycota bölümleri içinde yer alan üç sınıf, yedi takım, 25 familya ve 50 cinse ait 86 tür belirlenmiştir. Belirlenen taksonlar, habitatları, substratları, toplanma yerleri, coğrafi koordinatları ve toplayıcı numaraları ile birlikte listelenmiştir. Bunlardan bir tanesi, Melanophyllum haematospermum (Bull.: Fr.) Kreisel, Melanophyllum Velen. cinsinin ilk üyesi niteliğinde, Türkiye için yeni kayıttır.

Anahtar Kelimeler: Biyoçeşitlilik, yeni kayıt, makromantar, mikota, Türkiye

Citation: Çağli A, Öztürk A (2020). Macromycetes determined in Muradiye (Van) district. Anatolian Journal of Botany 4(1): 57-64.

\section{Introduction}

Fungi are an important group of organism for nature, and about 1.5 million species of them have been supposed to exist in the world (Hawksworth et al., 1995). Those fungi with fruiting bodies that can be seen by naked eye are known as macrofungi and almost 15.000 of them were determined in Europe (Lukić, 2008).

Starting especially from the first quarter of $20^{\text {th }}$ century, many studies have also been carried out on the macrofungi of Turkey and almost 2.400 taxa have been determined (Sesli and Denchev, 2014; Solak et al., 2015). But the macrofungal biodiversity of Turkey hasn't completely been determined yet.

Muradiye is a district of Van province within Eastern Anatolian region of Turkey and situated between $38^{\circ} 44^{\prime}$ $39^{\circ} 12^{\prime}$ north latitudes and $43^{\circ} 34^{\prime}-43^{\circ} 53^{\prime}$ east longitudes. The district is surrounded by Diyadin (Ağrı) district to north, central district of Van to the south, Çaldiran and Özalp districts to the east and Erciş district to the west (Figure 1). Bendimahi and Zincirli streams, and Van lake are the main hydrologic components of the region. According to the data obtained from Muradiye Meteorological Station, the region has an annual average temperature of $9.2{ }^{\circ} \mathrm{C}$ and a total precipitation of $582 \mathrm{~mm}$. Steppe vegetation is the dominant vegetation type in the region. Populus L., Salix L., Quercus L., Malus Mill. and Juglans L. are the major tree population affecting the growth of macrofungi in the region.

Though some macrofungal biodiversity studies have been carried out in neighboring districts and provinces (Demirel, 1996; Demirel and Uzun, 1996; Uzun and Demirel, 1996; Demirel et al., 2002, 2003, 2004, 2010, 2015; Kaya, 2000a,b,c,d; Kaya, 2001; Kaya and Demirel, 2000; Akçay et al., 2010; Demirel and Koçak, 2016; Şelem et al., 2019) there isn't a complete study on the macrofungi of Muradiye district.

The study aims to determine the macrofungi of the district and make a contribution to the mycobiota of Turkey.

\section{Materials and Method}

Macrofungi samples were collected from the region within the boundaries of Muradiye districts of Van province. They were photographed at their natural habitat and descriptive characteristics of them were noted. Then the samples were transferred to the laboratory and microscopic investigations were performed. The samples were identified with the help of the related references (Phillips, 1981; Moser, 1983; Buczacki, 1989; Bresnsky and Besl, 1990; Ellis and Ellis, 1990; Breitenbach and Kränzlin, 1984, 1986, 1991, 1995; 2000; Jordan, 1995; Wasser, 1996, 2000; Peterson et al., 2000; Kränzlin, 2005; Hausknecht, 2009; Amandeep et al., 2015). The samples are kept at the fungarium of Van Yüzüncü Yıl University (VANF).

\section{Results}

The determined taxa are listed in alphabetical order and the systematics follows Index Fungorum (accessed on 5 December 2019).

Ascomycota Whittaker

Leotiomycetes O.E. Erikss. \& Winka

Helotiales Nannf.

Helotiaceae Rehm

1. Hymenoscyphus calyculus (Fr.) W. Phillips

Muradiye district centre, on Salix sp. stumps, 38 ${ }^{\circ} 59^{\prime} 783^{\prime \prime} \mathrm{N}$, 4346'093"E, 1724 m, 24.10.2014, Çağli. 181; Değerbilir 
village, on Populus sp. stump, 39 $03^{\prime} 646^{\prime \prime} \mathrm{N}, 43^{\circ} 44^{\prime} 799^{\prime \prime} \mathrm{D}$, 1806 m, 24.10.2014, Çağli. 187.

Pezizomycetes O.E.Erikss. \& Winka

Pezizales J.Schröt.

Helvellaceae Fr.

2. Helvella leucopus Pers.

Around Muradiye Waterfall, on soil under Populus sp., $39^{\circ} 03^{\prime} 915^{\prime \prime} \mathrm{N}, 43^{\circ} 45^{\prime} 400^{\prime \prime} \mathrm{E}, 1828 \mathrm{~m}, 19.05 .2015$, Çağli. 209.

\section{Paxina queletii (Bres.) Stangl}

Değerbilir village, on soil under Populus sp., 390 $03^{\prime} 646^{\prime \prime} \mathrm{N}$,

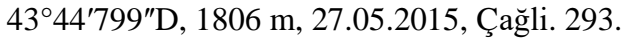

Morchellaceae Rchb.

4. Morchella elata Fr.

Değerbilir village, on soil under Populus sp., $39^{\circ} 03^{\prime} 732^{\prime \prime} \mathrm{N}$, 4345'169"E, 1827 m, 19.05.2015, Çağli. 239.

\section{Verpa bohemica (Krombh.) J. Schröt}

Muradiye District centre, on soil under Populus sp., $38^{\circ} 59^{\prime} 783^{\prime \prime} \mathrm{N}, 43^{\circ} 46^{\prime} 093^{\prime \prime} \mathrm{E}, 1724 \mathrm{~m}, 03.05 .2015$, Çağli. 206.

6. Verpa conica (O.F. Müll.) Sw.

Old Görecek village, on soil Populus sp., 3904'881"N, 4345'514"E, 1813 m, 19.05.2015, Çağli. 243.

\section{Pezizaceae Dumort.}

\section{Peziza domiciliana Cooke}

Küçükköy village, on soil under Populus sp. $38^{\circ} 59^{\prime} 473^{\prime \prime} \mathrm{N}$, $43^{\circ} 46^{\prime} 421^{\prime \prime} \mathrm{E}, 1667 \mathrm{~m}, 26.05 .2014$, Çăgli. 73.

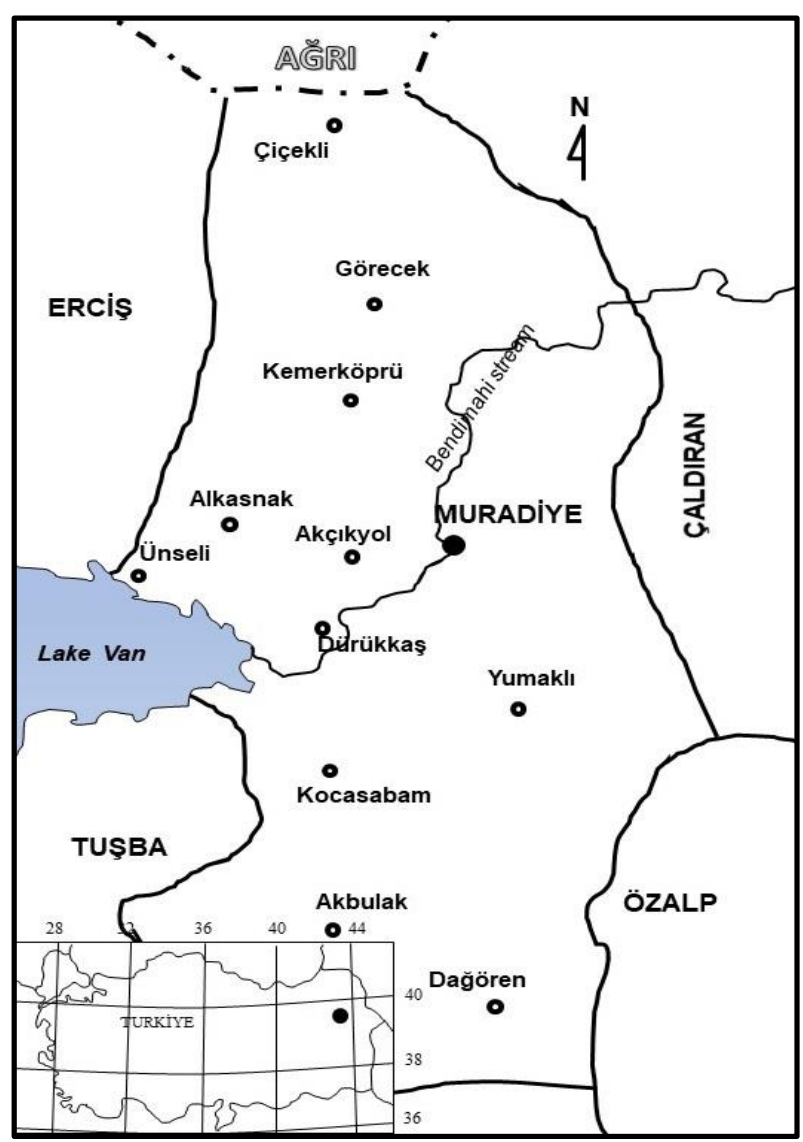

Figure 1. Map of the research area.

\section{Peziza praetervisa Bres}

Küçükköy village, on burned place under Salix sp., $38^{\circ} 59^{\prime} 473^{\prime \prime} \mathrm{N}, 43^{\circ} 46^{\prime} 421^{\prime \prime} \mathrm{E}, 1667$ m, 26.05.2014, Çağli. 82.

\section{Pyronemataceae Corda}

9. Geopora arenosa (Fuckel) S. Ahmad

Muradiye District centre, on soil under Populus sp., 38 $59^{\prime} 783^{\prime \prime} \mathrm{N}, 43^{\circ} 46^{\prime} 093^{\prime \prime} \mathrm{E}, 1724$ m, 26.05.2014, Çağli. 78.

\section{Scutellinia scutellata (L.) Lambotte}

Muradiye disrtict centre, on Salix and Populus sp. stumps, 38 59'783"N, 4346'093"E, 1724 m, 06.05.2014, Çağli. 36; Around Muradiye Waterfall, $39^{\circ} 03^{\prime} 913^{\prime \prime} \mathrm{N}, 43^{\circ} 45^{\prime} 400^{\prime \prime} \mathrm{E}$, 1828 m, 26.05.2014, Çağli. 95.

\section{Basidiomycota R.T.Moore}

Agaricomycetes Doweld

Agaricales Underw.

Agaricaceae Chevall.

11. Agaricus arvensis Schaeff.

Yumaklı village, on soil among grass under Populus sp., 38 $56^{\prime} 565^{\prime \prime} \mathrm{N}, 43^{\circ} 45^{\prime} 078^{\prime \prime} \mathrm{E}, 1639$ m 19.05.2015, Çağli. 221.

\section{Agaricus bernardii Quél}

Küçükköy village, meadow, 38 $59^{\prime} 473^{\prime \prime} \mathrm{N}, 43^{\circ} 46^{\prime} 421^{\prime \prime} \mathrm{E}$, 1667 m, 24.10.2014, Çağli. 178.

\section{Agaricus campestris L.}

Değerbilir village, meadow, $39^{\circ} 03^{\prime} 732^{\prime \prime} \mathrm{N}, 43^{\circ} 45^{\prime} 169^{\prime \prime} \mathrm{E}$, 1827 m, 20.10.2014, Çağli. 129.

\section{Agaricus pampeanus Speg.}

Görecek village, meadow, $39^{\circ} 03^{\prime} 520^{\prime \prime} \mathrm{N}, 43^{\circ} 45^{\prime} 517^{\prime \prime} \mathrm{E}$, 1865 m, 24.10.2014, Çağli. 165.

\section{Bovista plumbea Pers.}

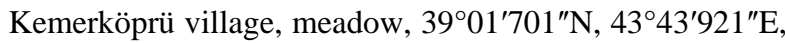
1822 m, 06.05.2014, Çağli. 17.

16. Coprinus comatus (O.F. Müll.) Pers.

Değerbilir village, meadow, $39^{\circ} 03^{\prime} 732^{\prime \prime} \mathrm{N}, 43^{\circ} 45^{\prime} 169^{\prime \prime} \mathrm{E}$, 1827 m, 19.05.2015, Çağli. 237.

\section{Lepiota cristata (Bolton) P. Kumm.}

Değerbilir village, among leaf litter under Populus sp. $39^{\circ} 03^{\prime} 732^{\prime \prime} \mathrm{N}, 43^{\circ} 45^{\prime} 169^{\prime \prime} \mathrm{E}, 1827 \mathrm{~m}, 20.10 .2014$, Çağli. 111.

\section{Lepiota helveola Bres.}

Değerbilir village, among leaf litter under Populus sp., 39 $03^{\prime} 732^{\prime \prime} \mathrm{N}, 43^{\circ} 45^{\prime} 169^{\prime \prime} \mathrm{E}, 1827 \mathrm{~m}$, Çă̆li. 116; Gönderme village, $39^{\circ} 05^{\prime} 152^{\prime \prime} \mathrm{N}, 43^{\circ} 49^{\prime} 028^{\prime \prime} \mathrm{E}, 1907 \mathrm{~m}, 20.10 .2014$, Çağli. 118.

\section{Leucoagaricus leucothites (Vittad.) Wasser}

Değerbilir village, among grass with needle litter, $39^{\circ} 03^{\prime} 732^{\prime \prime} \mathrm{N}, 43^{\circ} 45^{\prime} 169^{\prime \prime} \mathrm{E}, 1827 \mathrm{~m}, 20.10 .2014$, Çağli. 117.

\section{Lycoperdon pratense Pers.}

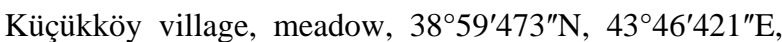
1667 m, 24.10.2014, Çağli. 179.

\section{Lycoperdon utriforme Bull.}

Değerbilir village, meadow, $39^{\circ} 03^{\prime} 732^{\prime \prime} \mathrm{N}, 43^{\circ} 45^{\prime} 169^{\prime \prime} \mathrm{E}$, 1827 m, 20.10.2014, Çağli. 131; Balaklı village, 
$38^{\circ} 58^{\prime} 507^{\prime \prime} \mathrm{N}, 43$ 44'083"E, 1695 m, 19.05.2015, Çağli. 236.

\section{Macrolepiota excoriata (Schaeff.) Wasser}

Görecek village, among grass, $39^{\circ} 03^{\prime} 520^{\prime \prime} \mathrm{N}, 43^{\circ} 45^{\prime} 517^{\prime \prime} \mathrm{E}$, 1865 m, 24.10.2014, Çağli. 166.

23. Melanophyllum haematospermum (Bull.: Fr.) Kreisel Pileus 12-24 $\mathrm{mm}$ in diameter, at first hemispherical to obtuse-conic, then campanulate later convex or applanate, with or without a small umbo; margin incurved when young, decurved to level at maturity, with greyish to greyish-brown veil fragments; surface cottony when young, suede-like to granulose, light to dingy-brown, darker at the center. Lamellae free to deeply adnexed, crowded, pinkishbrown, vinaceous-brown. Stipe 20-30 × 3.5-5 mm, cylindrical, straight to somewhat bent, enlarged to bulbous at the base, hollow or with a pith-like core in age, fragile, greyish, covered by reddish-brown to vinaceous-brown remnants of the universal veil (Figure 2a). Basidia 15-19 $\times$ 5.5-6.5 $\mu \mathrm{m}$, clavate to clavate-cylindrical, 4-spored. Spores 4.5-6.5 $\times$ 2.5-4 $\mu \mathrm{m}$, elliptic, oblong-elliptic to reniform, thin walled, smooth to minutely roughened, olive-brown (Figure 2b).

Melanophyllum haematospermum was reported to grow on humus-rich soil under both conifers and decidious trees as solitary or in groups (Wasser, 2000; Bujakiewicz, 2010).

Değerbilir village, on soil among leaf litter under Populus

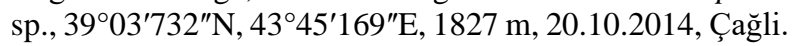
112.

\section{Bolbitiaceae Singer}

24. Conocybe semiglobata Kühner \& Watling

Küçükköy village, among grass, $38^{\circ} 59^{\prime} 473^{\prime \prime} \mathrm{N}$, 4346'421"E, 1667 m, 26.05.2014, Çağli. 46; Yakıncak village, $38^{\circ} 57^{\prime} 107^{\prime \prime} \mathrm{N}, 43^{\circ} 46^{\prime} 429^{\prime \prime} \mathrm{E}, 1660 \mathrm{~m}$, Çağli. 74.

\section{Conocybe siennophylla (Berk. \& Broome) Singer}

Muradiye District centre, among leaf litter under Populus sp., 38 $59^{\prime} 783^{\prime \prime} \mathrm{N}, 43^{\circ} 46^{\prime} 093^{\prime \prime} \mathrm{E}, 1724$ m, 26.05.2014, Çağli. 91; Durukkaş village, $38^{\circ} 57^{\prime} 391^{\prime \prime} \mathrm{N}, 43^{\circ} 40^{\prime} 898^{\prime \prime} \mathrm{E}, 1670 \mathrm{~m}$, 24.10.2014, Çăgli. 169

\section{Conocybe subovalis Kühner \& Watling}

Değerbilir village, meadow, $39^{\circ} 03^{\prime} 732^{\prime \prime} \mathrm{N}, 43^{\circ} 45^{\prime} 169^{\prime \prime} \mathrm{E}$, $1827 \mathrm{~m}$, 06.05.2014, Çağli. 16; Ayrancı village, $39^{\circ} 96^{\prime} 929^{\prime \prime} \mathrm{N}, 43^{\circ} 51^{\prime} 673^{\prime \prime} \mathrm{E}, 1937$ m, 26.05.2014, Çağli. 68; Küçükköy village, 38 $59^{\prime} 473^{\prime \prime} \mathrm{N}, 43^{\circ} 46^{\prime} 421^{\prime \prime} \mathrm{E}, 1667 \mathrm{~m}$, 20.10.2014, Çağli. 138.

\section{Conocybe tenera (Schaeff.) Fayod}

Küçükköy village, among grass under Populus sp., 38 $8^{\circ} 9^{\prime} 473^{\prime \prime} \mathrm{N}, 43^{\circ} 46^{\prime} 421^{\prime \prime} \mathrm{E}, 1667$ m, 26.05.2014, Çağli. 67; Around Muradiye Waterfall, $39^{\circ} 03^{\prime} 915^{\prime \prime} \mathrm{N}, 43^{\circ} 45^{\prime} 400^{\prime \prime} \mathrm{E}$, 1828 m, 19.05.2015, Çağli. 226.

\section{Conocybe velutipes (Velen.) Hauskn. \& Svrček}

Değerbilir village, among leaf litter under Populus sp., $39^{\circ} 03^{\prime} 732^{\prime \prime} \mathrm{N}, 43^{\circ} 45^{\prime} 169^{\prime \prime} \mathrm{E}, 1827 \mathrm{~m}, 20.10 .2014$, Çağli. 113 (Çağli et al., 2019).

\section{Galeropsis desertorum Velen. \& Dvořák}

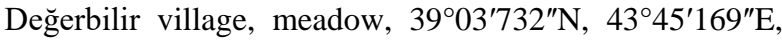
1827 m, 20.10.2014, Çağli. 144.

\section{Cortinariaceae R. Heim ex Pouzar}

30. Cortinarius vernus $H$. Lindstr. \& Melot

Değerbilir village, among leaf litter under Populus sp., 3903'732"N, 43ํ5'169"E, 1827 m, 06.05.2014, Çağli. 15; Around Muradiye Waterfall, $39^{\circ} 03^{\prime} 915^{\prime \prime} \mathrm{N}, 43^{\circ} 45^{\prime} 400^{\prime \prime} \mathrm{E}$, 1828 m, Çağli. 214; Muradiye District centre, $38^{\circ} 59^{\prime} 783^{\prime \prime} \mathrm{N}$, 4346'093"E, $1724 \mathrm{~m}$, Çağli. 223, Gönderme village, $39^{\circ} 05^{\prime} 152^{\prime \prime} \mathrm{N}, 43^{\circ} 49^{\prime} 028^{\prime \prime} \mathrm{E}, 1907 \mathrm{~m}, 19.05 .2015$, Çăgli. 229.

\section{Cyphellaceae Lotsy}

\section{Chondrostereum purpureum (Pers.) Pouzar}

Muradiye District centre, on Salix sp. stump, 38 $58^{\circ} 783^{\prime \prime} \mathrm{N}$, 4346'093"E, 1724 m, 24.10.2014, Çağli. 185.

\section{Entolomataceae Kotl. \& Pouzar}

32. Entoloma ameides (Berk. \& Broome) Sacc.

Görecek old village, meadow, $39^{\circ} 04^{\prime} 881^{\prime \prime} \mathrm{N}, 43^{\circ} 45^{\prime} 514^{\prime \prime} \mathrm{E}$, 1813 m, 19.05.2015, (Çağli et al., 2019).
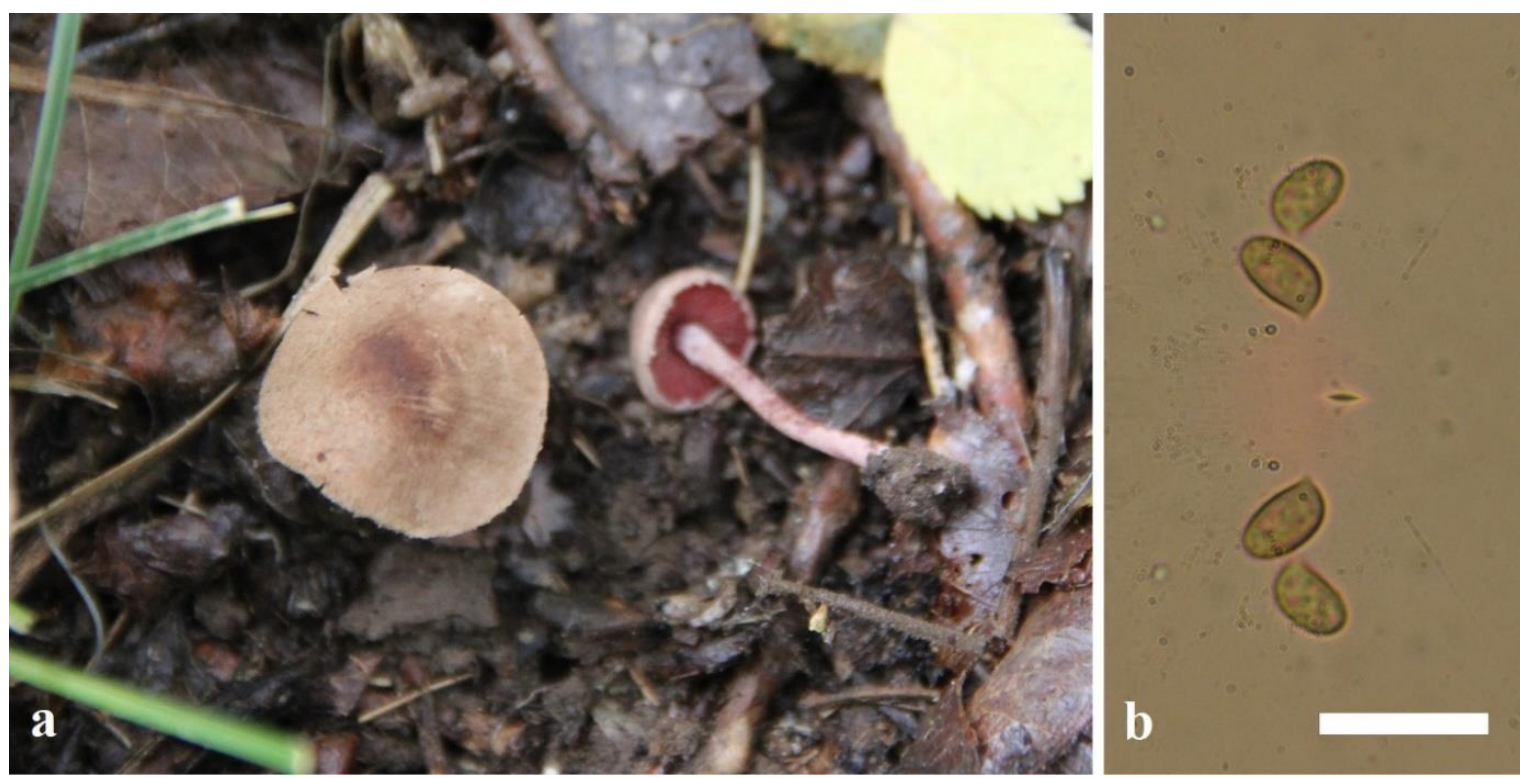

Figure 2. Basidiocarps (a) and basidiospores (b) of Melanophyllum haematospermum (bar: $10 \mu \mathrm{m}$ ). 


\section{Hymenogastraceae Vittad.}

\section{Hebeloma cavipes Huijsman}

Muradiye District centre, among leaf litter under Populus sp., 38 $59^{\prime} 783^{\prime \prime} \mathrm{N}, 43^{\circ} 46^{\prime} 093^{\prime \prime} \mathrm{E}, 1724$ m, 06.05.2014, Çağli. 35 .

\section{Hebeloma crustuliniforme (Bull.) Quél.}

Görecek village, among leaf litter under Populus sp., $39^{\circ} 03^{\prime} 520^{\prime \prime} \mathrm{N}, 4^{\circ} 45^{\prime} 517^{\prime \prime} \mathrm{E}, 1865 \mathrm{~m}, 20.10 .2014$, Çağli. 136; Muradiye district centre, $38^{\circ} 59^{\prime} 783^{\prime \prime} \mathrm{N}, 43^{\circ} 46^{\prime} 093^{\prime \prime} \mathrm{E}$, $1724 \mathrm{~m}$, Çağli. 155; Yumaklı village, 38056'565"N, 4345'078"E,1639 m, 24.10.2014, Çăgli. 164; Yakıncak village, $38^{\circ} 57^{\prime} 107^{\prime \prime} \mathrm{N}, 43^{\circ} 46^{\prime} 429^{\prime \prime} \mathrm{E}, 1660 \mathrm{~m}, 19.05 .2015$, Çağli. 224.

\section{Hebeloma pusillum J.E. Lange}

Değerbilir village, among leaf litter under Populus sp., $39^{\circ} 03^{\prime} 732^{\prime \prime} \mathrm{N}, 43^{\circ} 45^{\prime} 169^{\prime \prime} \mathrm{E}, 1827 \mathrm{~m}$, Şelale dere yanı; $39^{\circ}$ $03^{\prime} 913^{\prime \prime} \mathrm{N}, 43^{\circ} 45^{\prime} 400^{\prime \prime} \mathrm{E}, 1828 \mathrm{~m}, 20.10 .2014$, Çağli. 141, 142.

\section{Psilocybe coronilla (Bull.) Noordel.}

Çayırlık, Kemerköprü köyü; 3901'701'N, 4343'921"E, $1822 \mathrm{~m}$, 06.05.2014, Çağli. 21; Görecek village, $39^{\circ} 03^{\prime} 520^{\prime \prime} \mathrm{N}, 4^{\circ} 45^{\prime} 517^{\prime \prime} \mathrm{E}, 1865 \mathrm{~m}, 24.10 .2014$, Çağli. 168; Ayranc1 village, $39^{\circ} 06^{\prime} 405^{\prime \prime} \mathrm{K}, 43^{\circ} 50^{\prime} 567^{\prime \prime} \mathrm{E}, 1972 \mathrm{~m}$, Çağli. 242.

\section{Incertae Sedis}

37. Panaeolus papilionaceus (Bull.) Quél.

Muradiye district centre, on manured soil, $38^{\circ} 59^{\prime} 783^{\prime \prime} \mathrm{N}$, 4346'093"E, 1724 m, 26.05.2014, Çağli. 40; Küçükköy village, $38^{\circ} 59^{\prime} 473^{\prime \prime} \mathrm{N}, 43^{\circ} 46^{\prime} 421^{\prime \prime} \mathrm{E}, 1667 \mathrm{~m}, 24.10 .2014$, Çağli. 176; Gönderme village, $39^{\circ} 05^{\prime} 152^{\prime \prime} \mathrm{N}, 43^{\circ} 49^{\prime} 028^{\prime \prime} \mathrm{E}$, 1907 m, 27.05.2015, Çağli. 270.

\section{Inocybaceae Jülich}

38. Inocybe dulcamara (Pers.) P. Kumm

Muradiye District centre, among grass under Populus and Salix sp., 38 ${ }^{\circ} 59^{\prime} 783^{\prime \prime} \mathrm{N}, 43^{\circ} 46^{\prime} 093^{\prime \prime} \mathrm{E}, 1724 \mathrm{~m}, 03.05 .2014$, Çağli. 1; Küçükköy village, $38^{\circ} 59^{\prime} 473^{\prime \prime} \mathrm{N}, 43^{\circ} 46^{\prime} 421^{\prime \prime} \mathrm{E}$, $1667 \mathrm{~m}$, 03.06.2014, Çağli. 96; Yumaklı village, $38^{\circ} 56^{\prime} 565^{\prime \prime} \mathrm{N}, 43^{\circ} 45^{\prime} 078^{\prime \prime} \mathrm{E}, 1639 \mathrm{~m}, 03.05 .2015$, Çağli. 207.

\section{Inocybe flocculosa Sacc}

Değerbilir village, among grass under Populus sp., $39^{\circ} 03^{\prime} 732^{\prime \prime} \mathrm{N}, 4^{\circ} 45^{\prime} 169^{\prime \prime} \mathrm{E}, 1827 \mathrm{~m}, 20.10 .2014$, Çağli. 137; Around Muradiye Waterfall, $39^{\circ} 03^{\prime} 915^{\prime \prime} \mathrm{N}$, 4345'400"E, 1828 m, 19.05.2015, Çağli. 213.

40. Inocybe rimosa (Bull.) P. Kumm.

Populus sp. alt1, Görecek village, among grass, 3903'520"N, 4345'517"E, 1865 m, 26.05.2014, Çağli. 76; Muradiye District centre, 38 $59^{\prime} 783^{\prime \prime} \mathrm{N}, 43^{\circ} 46^{\prime} 093^{\prime \prime} \mathrm{E}, 1724$ $\mathrm{m}$, Çağli. 92; Yumaklı village, $38^{\circ} 56^{\prime} 565^{\prime \prime} \mathrm{N}, 43^{\circ} 45^{\prime} 078^{\prime \prime} \mathrm{E}$, $1639 \mathrm{~m}$, 03.06.2014, Çağli. 98; Yakıncak village, 38 ${ }^{\circ} 7^{\prime} 107^{\prime \prime} \mathrm{N}, 43^{\circ} 46^{\prime} 429^{\prime \prime} \mathrm{E}, 1660$ m, Çağli. 99; Keçikıran village, $38^{\circ} 58^{\prime} 252^{\prime \prime} \mathrm{N}, 43^{\circ} 38^{\prime} 170^{\prime \prime} \mathrm{E}, 1705 \mathrm{~m}$, Çağli. 100; Ünseli village, $38^{\circ} 58^{\prime} 821^{\prime \prime} \mathrm{N}, \quad 43^{\circ} 35^{\prime} 283^{\prime \prime} \mathrm{E}, 1667 \mathrm{~m}$, 20.10.2014, Çă̆li. 134

\section{Inocybe splendens R. Heim}

Populus sp., alt1, Şelale yanı; $39^{\circ} 03^{\prime} 915^{\prime \prime} \mathrm{N}, 43^{\circ} 45^{\prime} 400^{\prime \prime} \mathrm{E}$, 1828 m, 19.05.2015, Çağli. 231.

\section{Marasmiaceae Roze ex Kühner}

42. Marasmius oreades (Bolton) Fr.

Kemerköprü village, meadow, $39^{\circ} 01^{\prime} 701^{\prime \prime} \mathrm{N}, 43^{\circ} 43^{\prime} 921^{\prime \prime} \mathrm{E}$, $1822 \mathrm{~m}$, 06.05.2014, Çağli. 25; Küçükköy village, $38^{\circ} 59^{\prime} 473^{\prime \prime} \mathrm{N}, 43^{\circ} 46^{\prime} 421^{\prime \prime} \mathrm{E}, 1667 \mathrm{~m}$, Çağli. 275; Alkasnak village, $39^{\circ} 58^{\prime} 171^{\prime \prime} \mathrm{N}, 43^{\circ} 38^{\prime} 317^{\prime \prime} \mathrm{E}, 1702 \mathrm{~m}, 27.05 .2015$, Çağli. 288.

\section{Mycenaceae Roze}

\section{Mycena galericulata (Scop.) Gray}

Değerbilir village, on decaying Quercus sp. stump, 39 03'646"N, 4344'799"E, 1806 m, 06.05.2014, Çağli. 34; Muradiye District centre, 38 $59^{\prime} 783^{\prime \prime} \mathrm{N}, 43^{\circ} 46^{\prime} 093^{\prime \prime} \mathrm{E}, 1724$ m, 19.05.2015, Çağli. 259.

\section{Mycena niveipes (Murrill) Murrill}

Muradiye District centre, on decaying stump, 38 $59^{\prime} 783^{\prime \prime} \mathrm{N}$, 4346'093"E, 1724 m, 26.05.2014, Çağli. 88.

\section{Hemimycena delectabilis (Peck) Singer}

Around Muradiye Waterfall, on leaf litter under Populus sp., 3903'915"N, 43º45'400"E, 1828 m, 20.10.2014, Çağli. 114.

\section{Pleurotaceae Kühner \\ 46. Pleurotus eryngii (DC.) Quél.}

Around Muradiye Waterfall, on Eryngium sp. remains, $39^{\circ} 03^{\prime} 915^{\prime \prime} \mathrm{N}, 43^{\circ} 45^{\prime} 400^{\prime \prime} \mathrm{E}, 1828$ m, 06.05.2014, Çağli. 3.

\section{Pleurotus ostreatus (Jacq.) P. Kumm.}

Değerbilir village, on Populus and Salix sp. stumps, 39 03'732"N, 4345'169"E, 1827 m, 06.05.2014, Çağli. 11; Muradiye District centre, $38^{\circ} 59^{\prime} 783^{\prime \prime} \mathrm{N}, 43^{\circ} 46^{\prime} 093^{\prime \prime} \mathrm{E}, 1724$ $\mathrm{m}, 26.05 .2014$, Çağli. 89; Karahan village, $38^{\circ} 56^{\prime} 725^{\prime \prime} \mathrm{N}$, 4339'836"E, $1645 \mathrm{~m}$, Çağli. 105; Değerbilir village, 39 $03^{\prime} 646^{\prime \prime} \mathrm{N}, 43^{\circ} 44^{\prime} 799^{\prime \prime} \mathrm{E}, 1806 \mathrm{~m}, 20.10 .2014$, Çağli. 195 ; Kemerköprü village, $39^{\circ} 01^{\prime} 701^{\prime \prime} \mathrm{N}, 43^{\circ} 43^{\prime} 921^{\prime \prime} \mathrm{E}, 1822$ m, 27.10.2015, Çăgli. 263.

\section{Pluteaceae Kotl. \& Pouzar}

48. Pluteus salicinus (Pers.) P. Kumm.

Değerbilir village, on wood remains, $39^{\circ} 03^{\prime} 732^{\prime \prime} \mathrm{N}$, 4345'169"E, 1827 m, 20.10.2014, Çağli. 110; Yakıncak village, $38^{\circ} 57^{\prime} 107^{\prime \prime} \mathrm{N}, 43^{\circ} 46^{\prime} 429^{\prime \prime} \mathrm{E}, 1660 \mathrm{~m}, 24.10 .2014$, Çağli. 170.

49. Volvopluteus gloiocephalus (DC.) Vizzini, Contu \& Justo

Değerbilir village, among grass, $39^{\circ} 03^{\prime} 646^{\prime \prime} \mathrm{N}$, 4344'799"E, 1806 m, 19.05.2015, Çağli. 253.

Psathyrellaceae Vilgalys, Moncalvo \& Redhead 50. Coprinellus disseminatus (Pers.) J.E. Lange

Muradiye District centre, around Salix sp. stump, 38 $59^{\prime} 783^{\prime \prime} \mathrm{N}, 43^{\circ} 46^{\prime} 093^{\prime \prime E}, 1724$ m, 06.05.2014, Çağli. 28; Karahan village, $38^{\circ} 56^{\prime} 725^{\prime \prime} \mathrm{N}, 43^{\circ} 39^{\prime} 836^{\prime \prime} \mathrm{E}, 1645 \mathrm{~m}$ 03.06.2014, Çağli. 104; Balaklı village, $38^{\circ} 58^{\prime} 507^{\prime \prime} \mathrm{N}$, 4344'083"E, 1695 m 19.05.2015, Çağli. 220.

51. Coprinellus micaceus (Bull.) Vilgalys, Hopple \& Jacq. Johnson

Değerbilir village, around Salix sp. stump, 39 $03^{\prime} 732^{\prime \prime} \mathrm{N}$,

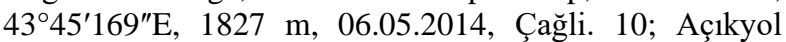

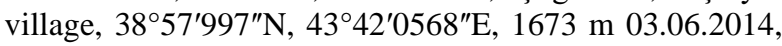
Çağli. 48; Muradiye District centre, $38^{\circ} 59^{\prime} 783^{\prime \prime} \mathrm{N}$, 
4346'093"E, 1724 m, 20.10.2014, Çağli. 102; Küçükköy village, $38^{\circ} 59^{\prime} 473^{\prime \prime} \mathrm{N}, 43^{\circ} 46^{\prime} 421^{\prime \prime} \mathrm{E}, 1667 \mathrm{~m}, 26.05 .2014$, Çağli. 132; Görecek village, $39^{\circ} 04^{\prime} 881^{\prime \prime} \mathrm{N}, 43^{\circ} 45^{\prime} 514^{\prime \prime} \mathrm{E}$, $1813 \mathrm{~m}, 19.05 .2015$, Çağli. 234; Around Muradiye Waterfall, 39 $03^{\prime} 915^{\prime \prime} \mathrm{N}, 43^{\circ} 45^{\prime} 400^{\prime \prime} \mathrm{E}, 1828 \mathrm{~m}, 27.05 .2015$, Çağli. 262.

52. Coprinopsis atramentaria (Bull.) Redhead, Vilgalys \& Moncalvo

Muradiye District centre, around decaying Populus sp. stump, 38 59'783"N, 4346'093"E, 1724 m, 26.05.2014, Çağli. 37; Değerbilir village, $39^{\circ} 03^{\prime} 732^{\prime \prime} \mathrm{N}, 43^{\circ} 45^{\prime} 169^{\prime \prime} \mathrm{E}$, $1827 \mathrm{~m}, 20.10 .2014$, Çağli. 151; Around Muradiye Waterfall, 39 $03^{\prime} 915^{\prime \prime} \mathrm{N}, 43^{\circ} 45^{\prime} 400^{\prime \prime} \mathrm{E}, 1828 \mathrm{~m}, 19.05 .2015$, Çăgli. 233.

53. Coprinopsis marcescibilis (Britzelm.) Örstadius \& E. Larss.

Görecek village, among leaf litter, $39^{\circ} 03^{\prime} 520^{\prime \prime} \mathrm{N}$, 4345'517"E, $1865 \mathrm{~m}$, Çağli. 167; Küçükköy village, $38^{\circ} 59^{\prime} 473^{\prime \prime} \mathrm{N}, 43^{\circ} 46^{\prime} 421^{\prime \prime} \mathrm{E}, 1667 \mathrm{~m}, 24.10 .2014$, Çağli. 177 ; Around Muradiye Waterfall, $39^{\circ} 03^{\prime} 915^{\prime \prime} \mathrm{N}$, 4345'400"E, 1828 m, 19.05.2015, Çağli. 235.

\section{Coprinopsis patouillardii (Quél.) G. Moreno}

Muradiye District centre, on manured soil, $38^{\circ} 59^{\prime} 783^{\prime \prime} \mathrm{N}$, 43 46'093"E, 1724 m, 20.10.2014, Çağli. 156.

55. Parasola plicatilis (Curtis) Redhead, Vilgalys \& Hopple

Küçükköy village, on manured soil among grass, 38 $59^{\prime} 473^{\prime \prime} \mathrm{N}, 43^{\circ} 46^{\prime} 421^{\prime \prime} \mathrm{E}, 1667$ m, 26.05.2014, Çağli. 79.

56. Psathyrella bipellis (Quél.) A.H. Sm.

Görecek village, among grass under Populus sp., $39^{\circ} 04^{\prime} 881^{\prime \prime} \mathrm{N}, 43^{\circ} 45^{\prime} 514^{\prime \prime} \mathrm{E}, 1813 \mathrm{~m}, 20.10 .2014$, Çağli. 147.

\section{Psathyrella candolleana (Fr.) Maire}

Muradiye District centre, around Populus sp. stump, 38 $59^{\prime} 783^{\prime \prime} \mathrm{N}, 43^{\circ} 46^{\prime} 093^{\prime \prime} \mathrm{E}, 1724$ m, 26.05.2014, Çağli. 43; around Muradiye Waterfall, $39^{\circ} 03^{\prime} 915^{\prime \prime} \mathrm{N}, 43^{\circ} 45^{\prime} 400^{\prime \prime} \mathrm{E}$, 1828 m, 20.10.2014, Çağli. 119; Değerbilir village, $39^{\circ} 03^{\prime} 732^{\prime \prime} \mathrm{N}, 43^{\circ} 45^{\prime} 169^{\prime \prime} \mathrm{E}, 1827 \mathrm{~m}$, Çağli. 123; Yumaklı village, 38 $56^{\prime} 565^{\prime \prime} \mathrm{N}, 4^{\circ} 45^{\prime} 078^{\prime \prime} \mathrm{E}, 1639 \mathrm{~m}$, Çağli. 203; Küçükköy village, $38^{\circ} 59^{\prime} 473^{\prime \prime} \mathrm{N}, 43^{\circ} 46^{\prime} 421^{\prime \prime} \mathrm{E}, 1667 \mathrm{~m}$, 27.05.2015, Çağli. 264.

\section{Psathyrella corrugis (Pers.) Konrad \& Maubl.}

Muradiye District centre, among leaf litter under Populus sp., 38 $59^{\prime} 783^{\prime \prime} \mathrm{N}, 43^{\circ} 46^{\prime} 093^{\prime \prime} \mathrm{E}, 1724$ m, 19.05.2015, Çağli. 257.

\section{Psathyrella microrhiza (Lasch) Konrad \& Maubl.}

Değerbilir village, among leaf litter under Populus sp., $39^{\circ} 03^{\prime} 732^{\prime \prime} \mathrm{N}, 43^{\circ} 45^{\prime} 169^{\prime \prime} \mathrm{E}, 1827 \mathrm{~m}, 20.10 .2014$, Çağli. 122.

\section{Psathyrella prona (Fr.) Gillet}

Populus sp. alt1, Merkez; 38 59'783"N, 43 46'093"E, 1724 $\mathrm{m}, 26.05 .2014$, Görece eski köy; $39^{\circ} 04^{\prime} 881^{\prime \prime} \mathrm{N}, 43^{\circ}$ 45'514"E, 1813 m, Balaklı köyü; $38^{\circ} 58^{\prime} 507^{\prime \prime} \mathrm{N}, 43^{\circ}$ 44'083"E, 1695 m, 19.05.2015, ÇAĞLİ. 41, 222, 244.

61. Psathyrella pseudogracilis (Romagn.) M.M. Moser

Muradiye District centre, on decaying stump, 38 ${ }^{\circ} 59^{\prime} 783^{\prime \prime} \mathrm{N}$, 434'093"E, 1724 m, 24.10.2014, Çağli. 186.

\section{Psathyrella spadiceogrisea (Schäeff.) Maire}

Küçükköy village, on decaying stump, 38 $59^{\prime} 473^{\prime \prime} \mathrm{N}$,

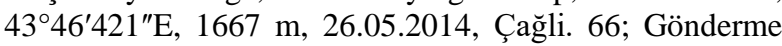
village, $39^{\circ} 05^{\prime} 152^{\prime \prime} \mathrm{N}, 43^{\circ} 49^{\prime} 028^{\prime \prime} \mathrm{E}, 1907 \mathrm{~m}, 24.10 .2014$, Çağli. 175.

Strophariaceae Singer \& A.H. Sm. 63. Agrocybe dura (Bolton) Singer

Görecek village, meadow, $39^{\circ} 03^{\prime} 520^{\prime \prime} \mathrm{N}, 43^{\circ} 45^{\prime} 517^{\prime \prime} \mathrm{E}$, $1865 \mathrm{~m}$, 06.05.2014, Çağli. 7; Kemerköprü village, $39^{\circ} 01^{\prime} 701^{\prime \prime} \mathrm{N}, 43^{\circ} 43^{\prime} 921^{\prime \prime} \mathrm{E}, 1822 \mathrm{~m}, 06.05 .2014$, Çağli. 18; Ünseli village, $38^{\circ} 58^{\prime} 821^{\prime \prime} \mathrm{N}, 43^{\circ} 35^{\prime} 283^{\prime \prime} \mathrm{E}, 1667 \mathrm{~m}$, 26.05.2014, Çağli. 45.

64. Agrocybe paludosa (J.E. Lange) Kühner \& Romagn. ex Bon

Muradiye District centre, meadow, $38^{\circ} 59^{\prime} 783^{\prime \prime} \mathrm{N}$, 4346'093"E, 1724 m, 06.05.2014, Çağli. 30.

\section{Agrocybe pediades (Fr.) Fayod}

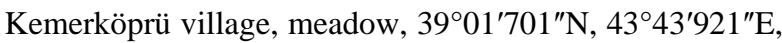
$1822 \mathrm{~m}$, 06.05.2014, Çağli. 20; Keçikıran village, $38^{\circ} 58^{\prime} 252^{\prime \prime} \mathrm{N}, 43^{\circ} 38^{\prime} 170^{\prime \prime} \mathrm{E}, 1705$ m 26.05.2014; Çağli. 42; Alkasnak village, $39^{\circ} 58^{\prime} 171^{\prime \prime} \mathrm{N}, 43^{\circ} 38^{\prime} 317^{\prime \prime} \mathrm{E}, 1702 \mathrm{~m}$ 19.05.2015, Çağli. 238.

\section{Agrocybe praecox (Pers.) Fayod}

Muradiye District centre, among grass, $38^{\circ} 59^{\prime} 783^{\prime \prime} \mathrm{N}$, 4346'093"E, $1724 \mathrm{~m}$, Çağli. 44; Küçükköy village, $38^{\circ} 59^{\prime} 473^{\prime \prime} \mathrm{N}, 43^{\circ} 46^{\prime} 421^{\prime \prime} \mathrm{E}, 1667 \mathrm{~m}, 26.05 .2014$, Çağli. 55 ; around Muradiye Waterfall, $39^{\circ} 03^{\prime} 915^{\prime \prime} \mathrm{N}, 43^{\circ} 45^{\prime} 400^{\prime \prime} \mathrm{E}$, 1828 m, 19.05.2015, Çağli. 208.

\section{Agrocybe vervacti (Fr.) Singer}

Kemerköprü village, among leaf litter under Populus sp., $39^{\circ} 01^{\prime} 701^{\prime \prime} \mathrm{N}, 43^{\circ} 43^{\prime} 921^{\prime \prime} \mathrm{E}, 1822 \mathrm{~m}$, Çağli. 23; Değerbilir village, $39^{\circ} 03^{\prime} 646^{\prime \prime} \mathrm{N}, 43^{\circ} 44^{\prime} 799^{\prime \prime} \mathrm{E}, 1822 \mathrm{~m}, 06.05 .2014$, Çağli. 24.

\section{Cyclocybe cylindracea (DC.) Vizzini \& Angelini}

Muradiye District centre, around Populus sp. stump, $38^{\circ} 59^{\prime} 783^{\prime \prime} \mathrm{N}, 4^{\circ} 46^{\prime} 093^{\prime \prime} \mathrm{E}, 1724$ m, 24.10.2014, Çağli. 192.

69. Deconica merdicola (Huijsman) Noordel.

Değerbilir village, on manure, 3903'646"N, 4344'799" E, 1806 m, 20.10.2014, Çağli. 152.

70. Hypholoma fasciculare (Huds.) P. Kumm.

Muradiye District centre, around Salix sp. stump, 38 $59^{\prime} 783^{\prime \prime} \mathrm{N}, 43^{\circ} 46^{\prime} 093^{\prime \prime} \mathrm{E}, 1724$ m, 06.05.2014, Çağli. 33.

\section{Pholiota aurivella (Batsch) P. Kumm.}

Küçükköy village, on Salix sp. trunk, 3859'473"N, 43 46'421"E, $1667 \mathrm{~m}, 26.05 .2014$, Çağli. 80; Değerbilir village, $39^{\circ} 03^{\prime} 732^{\prime \prime} \mathrm{N}, 43^{\circ} 45^{\prime} 169^{\prime \prime} \mathrm{E}, 1827 \mathrm{~m}, 20.10 .2014$, Çağli. 135.

\section{Pholiota conissans (Fr.) M.M. Moser}

Küçükköy village, among grass around Populus sp. stump, $38^{\circ} 59^{\prime} 473^{\prime \prime} \mathrm{N}, 43^{\circ} 46^{\prime} 421^{\prime \prime} \mathrm{E}, 1667$ m, 26.05.2015, Çağli. 81.

\section{Pholiota gummosa (Lasch) Singer}

Değerbilir village, around Salix sp. stump, 39 $03^{\prime} 646^{\prime \prime} \mathrm{N}$, 43⒋'799"E, 1806 m, Çağli. 149; Karahan village, $38^{\circ} 56^{\prime} 725^{\prime \prime} \mathrm{N}, 43^{\circ} 39^{\prime} 836^{\prime \prime} \mathrm{E}, 1645 \mathrm{~m}, 20.10 .2014$, Çağli. 150. 
Tricholomataceae R. Heim ex Pouzar

74. Melanoleuca exscissa (Fr.) Singer

Değerbilir village, among grass, $39^{\circ} 03^{\prime} 646^{\prime \prime} \mathrm{N}$, 434ㄴ'799"E, 1806 m, 19.05.2015, Çağli. 241.

\section{Tricholoma populinum J.E. Lange}

Görecek village, under Populus sp., 39 $03^{\prime} 520^{\prime \prime} \mathrm{N}$, 4345'517"E, 1865 m, 24.10.2014, Çağli. 163.

76. Tricholoma scalpturatum (Fr.) Quél.

Değerbilir village, under Populus sp., $39^{\circ} 03^{\prime} 732^{\prime \prime} \mathrm{N}$, 4345'169"E, 1827 m, 20.10.2014, Çağli. 154.

\section{Lepista irina (Fr.) H.E. Bigelow}

Muradiye District centre, under Populus sp., 38 59'783"N, 4346'093"E, 1724 m, 26.05.2014, Çağli. 90.

\section{Lepista nuda (Bull.) Cooke}

Muradiye District centre, under Populus sp., 38 59'783"N, 4346'093"E, 1724 m, 24.10.2014, Çağli. 189.

\section{Lepista personata (Fr.) Cooke}

Değerbilir village, under Populus sp., $39^{\circ} 03^{\prime} 732^{\prime \prime} \mathrm{N}$, 4345'169"E, $1827 \mathrm{~m}, 03.06 .2014$, Çağli. 13; Muradiye District centre, $38^{\circ} 59^{\prime} 783^{\prime \prime} \mathrm{N}, 43^{\circ} 46^{\prime} 093^{\prime \prime} \mathrm{E}, 1724 \mathrm{~m}$, 06.05.2014, Çağli. 31; Açıkyol village, 38 ${ }^{\circ} 7^{\prime} 997^{\prime \prime} \mathrm{N}, 43^{\circ}$ 42'0568"E, 1673 m, 24.10.2014, Çağli. 188; Durukkaş village, 38 57'391"N, 43 40'898"E, 1670 m, Çağli. 258.

\section{Tubariaceae Vizzini}

80. Tubaria furfuracea (Pers.) Gillet

Muradiye District centre, on soil among leaf litter, 38 59'783"N, 4346'093"E, 1724 m, 06.05.2014, Çağli. 29; Küçükköy village, $38^{\circ} 59^{\prime} 473^{\prime \prime} \mathrm{N}, 43^{\circ} 46^{\prime} 421^{\prime \prime} \mathrm{E}, 1667 \mathrm{~m}$, Çağli. 53; Yumaklı village, $38^{\circ} 56^{\prime} 565^{\prime \prime} \mathrm{N}$, 43ํ5'078"E, 1639 m, 26.05.2014, Çağli. 54.

\section{Boletales E.-J. Girbert}

Paxillaceae Lotsy

81. Paxillus involutus (Batsch) Fr.

Değerbilir village, among grass under Populus sp., $39^{\circ} 03^{\prime} 732^{\prime \prime} \mathrm{N}, 43^{\circ} 45^{\prime} 169^{\prime \prime E}, 1827$ m, Çağli. 125; Karahan village, $38^{\circ} 56^{\prime} 725^{\prime \prime} \mathrm{N}, 43^{\circ} 39^{\prime} 836^{\prime \prime} \mathrm{E}, 1645 \mathrm{~m}, 20.10 .2014$, Çağli. 145.

\section{Hymenochaetales Oberw.}

\section{Hymenochaetaceae Donk}

82. Phellinus igniarius (L.) Quél.

Küçükköy village, on Salix sp. trunk, 38 59'473"N, 43ํ6'421"E, 1667 m, 26.05.2014, Çağli. 83.

\section{Polyporales Gäum.}

Polyporaceae Fr. ex Corda

\section{Cerioporus squamosus (Huds.) Quél.}

Değerbilir village, on Populus sp. stump, $39^{\circ} 03^{\prime} 732^{\prime \prime} \mathrm{N}$, 4345'169"E, $1827 \mathrm{~m}, 06.05 .2014$, Çağli. 14; Yakıncak village, $38^{\circ} 57^{\prime} 107^{\prime \prime} \mathrm{N}, 43^{\circ} 46^{\prime} 429^{\prime \prime} \mathrm{E}, 1660 \mathrm{~m}, 19.05 .2015$, Çağli. 219.

84. Lentinus tigrinus (Bull.) Fr.

Muradiye District centre, around Salix sp. stump, $38^{\circ} 59^{\prime} 783^{\prime \prime} \mathrm{N}, 43^{\circ} 46^{\prime} 093^{\prime \prime} \mathrm{E}, 1724 \mathrm{~m}, 06.05 .2014$, Çağli. 27; 26.05.2014, Çağli. 87; 24.10.2014, Çağli. 191; Küçükköy village, $38^{\circ} 59^{\prime} 473^{\prime \prime} \mathrm{N}, 43^{\circ} 46^{\prime} 421^{\prime \prime} \mathrm{E}, 1667 \mathrm{~m}, 19.05 .2015$, Çăgli. 260.

\section{Polyporus rhizophilus Pat.}

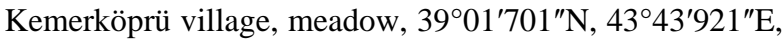
1822 m, 06.05.2014, Çağli. 22.

Russulales Kreisel ex P.M. Kirk, P.F. Cannon \& J.C. David Russulaceae Lotsy

86. Lactarius controversus Pers.

Değerbilir village, in poplar grove, $39^{\circ} 03^{\prime} 732^{\prime \prime} \mathrm{N}$, 4345'169"E, 1827 m 20.10.2014, Çağli. 146; Muradiye District centre, 38 $59^{\prime} 783^{\prime \prime} \mathrm{N}, 4^{\circ} 46^{\prime} 093^{\prime \prime} \mathrm{E}, 1724 \mathrm{~m}$, 24.10.2014, Çağli. 190.

\section{Discussions}

Eighty six macrofungi species belonging to 50 genera, 25 families, seven orders and three classes were determined from Muradiye district of Van province. Ten of the determined taxa belong to Ascomycota (1 Helotiales, 9 Pezizales) while 76 belong to Basidiomycota (70 Agaricales, 1 Boletales, 1 Hymenochaetales, 3 Polyporales, 1 Russulales).

The taxa are distributed in 25 families. Agaricaceae and Psathyrellaceae were found to the most crowded families each with 13 taxa. Strophariaceae is the second crowded one with 11 taxa. Two of the families (Bolbitiaceae, Tricholomataceae) comprises 6, two of them (Hymenogastraceae, Inocybaceae) comprises 4, three of them (Morchellaceae, Mycenaceae, Polyporaceae) comprises 3 taxa, five of them (Helvellaceae, Pezizaceae, Pyronemataceae, Pleurotaceae, Pluteaceae) comprises 2 taxa, and the rest of the 10 families comprises 1 taxon.

Thirty five (\%40.79) of the determined taxa are edible. But only three of them are collected and consumed by local people. Among them Agrocybe dura is known as "çimen mantarl" in the region while Pleurotus ostreatus is known as "ağaç/kavak mantarı" and Pleurotus eryngii is known as "heliz mantarı". Forty two (\%48.84) of them are inedible and $9(\% 10.47)$ of them are more or less poisonous.

The taxa determined in Muradiye district were compared with the findings of the studies carried out in neighbouring regions and some similarities were observed. These studies and the similarity percentages are given in Table 1.

Table 1. Similarity percentages of neighbouring studies with Muradiye district.

\begin{tabular}{lccc}
\hline Neighbouring study & $\begin{array}{c}\text { \# of iden- } \\
\text { tical taxa }\end{array}$ & $\begin{array}{c}\text { Total } \\
\text { taxa }\end{array}$ & $\begin{array}{c}\text { Similarity } \\
(\boldsymbol{\%})\end{array}$ \\
\hline Demirel (1996) & 18 & 50 & 36.00 \\
Demirel et al. (2002) & 23 & 45 & 51.11 \\
Demirel et al. (2003) & 23 & 114 & 20.18 \\
Demirel et al. (2004) & 17 & 162 & 10.49 \\
Akçay et al. (2010) & 20 & 50 & 40.00 \\
Demirel \& Koçak (2016) & 46 & 96 & 47.92 \\
Demirel et al. (2017) & 21 & 172 & 12.21 \\
\hline
\end{tabular}

Among the determined taxa, Bovista plumbea, Coprinus comatus, Psilocybe coronilla, Volvopluteus gloiocephalus and Polyporus rhizophilus were also recorded from the region by Demirel (1996). All the other taxa are new for the region while Melanophyllum haematospermum are new for Turkish mycobiota at genus level. 


\section{Acknowledgments}

The authors would like to thank Van Yüzüncü Yıl
University Research Project Unit (Project No: 2014-FBEYL-089) for its financial support.

\section{References}

Akçay ME, Uzun Y, Kaya A (2010). Malazgirt (Muş) yöresi makrofunguslarına katkılar. Mantar Dergisi 1(1): 14-20.

Amandeep K, Atri NS, Munruchi K (2015). Diversity of species of the genus Conocybe (Bolbitiaceae, Agaricales) collected on dung from Punjab, India. Mycosphere 6(1): 19-42.

Breitenbach J, Kränzlin F (1984). Fungi of Switzerland, Vol.1. Lucerne: Verlag Mykologia.

Breitenbach J, Kränzlin F (1986). Fungi of Switzerland, Vol.2. Lucerne: Verlag Mykologia.

Breitenbach J, Kränzlin F (1991). Fungi of Switzerland, Vol.3. Lucerne: Verlag Mykologia.

Breitenbach J, Kränzlin F (1995). Fungi of Switzerland, Vol.4. Lucerne: Verlag Mykologia.

Breitenbach J, Kränzlin F (2000). Fungi of Switzerland, Vol.5. Lucerne: Verlag Mykologia.

Bresnsky A, Besl H (1990). A color atlas of poisonous fungi. London: Wolfe Publishing.

Buczacki S (1989). Mushrooms and toadstools of Britain and Europe. Glasgow: Harper Collins Publishers.

Bujakiewicz A (2010). On some agarics occurring in carr forests. Acta Mycologia 45(1): 73-89.

Demirel K (1996). Van yöresi makrofungusları. Turkish Journal of Botany 20: 165-169.

Demirel K, Erdem Ö, Uzun Y, Kaya A (2010). Macrofungi of Hatila Valley National Park (Artvin-Turkey). Turkish Journal of Botany 34: 457-465.

Demirel K, Kaya A, Uzun Y (2003). Macrofungi of Erzurum province. Turkish Journal of Botany 27: 29-36.

Demirel K, Koçak MZ (2016). Zilan Vadisi'nin (Erciş-Van) makrofungal çeşitliliği. Mantar Dergisi 7(2): 122-134.

Demirel K, Uzun Y (1996). Van Gölü çevresinde belirlenen bazı odun tahripçisi makrofunguslar. Ekoloji ve Çevre Dergisi 21: $32-$ 36.

Demirel K, Uzun Y, Akçay ME, Keleş A, Acar İ, Efe V (2015). Van yöresi makromantarlarına katkılar. Mantar Dergisi 6(2): $12-$ 23.

Demirel K, Uzun Y, Biber G (2004). Macrofungi of Şavşat (Artvin) district. The Herb Journal of Systematic Botany 2: 191-206.

Demirel K, Uzun Y, Kaya A (2002). Macrofungi of Ağr1 Province. Turkish Journal of Botany 26: 291-295.

Demirel K, Uzun Y, Kaya A (2004). Some poisonous fungi of East Anatolia. Turkish Journal of Botany 28: 215-219.

Demirel K, Uzun Y, Keleş A, Akçay M, Acar İ (2017). Macrofungi of Karagöl-Sahara National Park (Şavşat-Artvin/Turkey). Biological Diversity and Conservation 10: 32-40.

Ellis BM, Ellis PJ (1990). Fungi without gills (Hymenomycetes and Gasteromycetes): An Identification Handbook. London: Chapman and Hall.

Hausknecht A, Kalameees K, Knudsen H, Mukhin V (2009). The genera Conocybe and Pholiotina (Agaricomycotina, Bolbitiaceae) in temperate Asia. Folia Cryptog. Estonica 45: 23-47.

Hawksworth DL, Kirk PM, Sutton BC, Pegler DN (1995). Ainsworth and Biby's dictionary of the fungi (8th Ed.). Wallingford: $\mathrm{CAB}$ International.

Index Fungorum. (2019). http://www.indexfungorum.org/names/Names.asp / [20 December 2019].

Jordan M (1995). The encyclopedia of fungi on Britain and Europe. Devon: David \& Charles Book.

Kaya A (2000a). Muş ve Bitlis yörelerinde tespit edilen yenen makrofunguslar, Türkiye VI. Yemeklik Mantar Kongresi Bildirileri 2-22 Eylül 2000, Bergama. s. 112-115.

Kaya A (2000b). New records of Tricholomataceae for the Mycoflora of Turkey. Bulletin of Pure and Applied Sciences, 19B: 7781.

Kaya A (2000c). Poisonous macrofungi determined in Muş and Bitlis provinces (Turkey), In: Özhatay, N., Plants of the Balkan Peninsula: into the next Millennium, Proceedings of the 2nd Balkan Botanical Congress, May, 2000, İstanbul - Turkey, Vol 1, Pp. 263-266.

Kaya A (2000d). Two New Genus Records for the Mycoflora of Turkey. Turkish Journal of Botany 24: 285-288.

Kaya A (2001). Contributions to the macrofungi flora of Bitlis province, Turkish Journal of Botany 25: 379-383.

Kaya A, Demirel K (2000). New additions to Turkish Entolomataceae. Hacettepe Bulletin of Natural Sciences and Engineering, Series A 28: 39-43.

Kränzlin F (2005). Fungi of Switzerland, Vol.6. Lucerne: Verlag Mykologia.

Lukić M (2008). The distribution and diversity of Boletus genus in central Serbia. Kragujevac Journal of Science 31: 59-68.

Moser M (1983). Keys to agarics and boleti (Polyporales, Boletales, Agaricales, Russulales). Stuttgart: Gustav Fischer Verlag.

Peterson KR, Desjardin DE, Hemmes DE (2000). Agaricales of the Hawaiian Islands. 6. Agaricaceae I. Agariceae: Agaricus and Melanophyllum. Sydowia 52(2): 204-257.

Phillips R (1981). Mushrooms and other fungi of Great Britain, Europe. London: Pan Books. 
Çağli \& Öztürk-Macromycetes determined ...

Sesli E, Denchev CM (2014). Checklists of the myxomycetes, larger ascomycetes, and larger basidiomycetes in Turkey. 6th edn. Mycotaxon Checklists Online (http,//www.mycotaxon.com/resources/checklists/sesli-v106-checklist.pdf): 1-136.

Solak MH, Işıloğlu M, Kalmış E, Allı H (2015). Macrofungi of Turkey, Checklist. Volume-II. Bornova: Üniversiteliler Ofset.

Şelem E, Keleş A, Acar İ, Demirel K (2019). Edible macrofungi determined in Gürpınar (Van) district. Anatolian Journal of Botany 3(1): 7-12.

Uzun Y, Demirel K (1996). Sarıkamış (Kars) yöresinin makrofunguslarına katkılar. Hacettepe Fen ve Mühendislik Bilimleri Dergisi 17: 121-132.

Wasser SP (1996). New, remarkable and rare higher Basidiomycetes from Israel. Czech Mycology 48(4): 237-250.

Wasser SP (2000). A contribution to the taxonomy and species diversity of the Agariceae tribe (Higher Basidiomycetes) of Israel mycobiota. Flora Mediterranea 10: 191-221. 